\title{
The new UK SARS-CoV-2 variant and lockdown - causes and consequences
}

\author{
Authors: Daniel Pan, ${ }^{A}$ Nadeesha Lakmal Mudalige, ${ }^{B}$ Shirley Sze, ${ }^{C}$ David Koeckerling, ${ }^{D}$ Oluwatobiloba Oyefeso, ${ }^{\mathrm{E}}$ \\ Joseph Barker, ${ }^{\mathrm{F}}$ Caroline ML Williams, ${ }^{\mathrm{G}}$ Julian W Tang ${ }^{\mathrm{H}}$ and Manish Pareek ${ }^{\mathrm{I}}$
}

The new variant of concern (VOC), B.1.1.7, has a distinct set of mutations in nucleotides encoding the spike (S) protein on the surface of SARS-CoV-2. SARS-CoV-2 previously accumulated mutations at a much slower rate, of 1-2 per month; the sudden appearance of a large cluster of mutations was thought to be unusual. We now suspect that VOC may have arisen from immunosuppressed individuals who shed virus for longer periods. Epidemiological analyses estimate VOC to be more infectious; this is of most concern because these estimates were calculated during periods where many regions of the UK were in high social distancing restrictions. Therefore, the previous 'tiered' system implemented in the UK was ineffective at containing VOC. The most likely reason for this is that previous restrictions, no matter how strict, still allowed for gatherings in certain places. VOC also has implications for the national vaccination programme - a higher proportion of people will need to be vaccinated with a more infectious virus. Prolongation of the second dose of vaccines to increase vaccine uptake has understandably caused concern, but is based on sound immunological principles. There is now an urgent need to monitor the effect of new variants on vaccine efficacy marking a new chapter in the global fight against COVID-19.

KEYWORDS: SARS-CoV-2, new variant, lockdown, vaccination, immunosuppression

DOI: 10.7861/clinmed.2021-0019

Authors: ANIHR academic clinical fellow in infectious diseases, University of Leicester, Leicester, UK and University Hospitals of Leicester NHS Trust, UK; ${ }^{B}$ Mudalige NIHR academic clinical fellow in paediatrics, London School of Tropical Medicine and Hygiene, London, UK; ' NIHR academic clinical fellow in cardiology, University of Leicester, Leicester, UK; Dacademic foundation doctor, University of Oxford, Oxfordshire, UK; Egeneral practitioner, Royal Free London NHS Foundation Trust, London, UK; FNIHR academic clinical fellow in cardiology, University of Leicester, Leicester, UK; ${ }^{\mathrm{G}}$ specialist registrar in infectious diseases and microbiology, University of Leicester, Leicester, UK; ${ }^{H}$ consultant virologist and honorary associate professor, University Hospitals of Leicester NHS Trust, UK; ${ }^{\mathrm{I}}$ associate professor and honorary professor in infectious diseases, University of Leicester, Leicester, UK and University Hospitals of Leicester NHS Trust, UK
Introduction: emergence of SARS-CoV-2 variants of concern

In December 2020, a new variant of SARS-CoV-2 was discovered by the COVID-19 Genomics United Kingdom Consortium (COGUK), called B.1.1.7 or variant of concern (VOC) 202012/01.' On sequencing analysis, 17 mutation sites were characterised in the new variant, compared to its most recent ancestor. ${ }^{1}$ Many of these mutations were in nucleotides encoding the spike (S) protein on the surface of SARS-CoV-2, through which the virus binds to and enters human cells. The concomitant increase in COVID-19 incidence due to VOC in the UK suggests that this new variant will quickly become the most dominant. ${ }^{2}$

SARS-COV-2 previously accumulated mutations at a relatively consistent rate, equating to approximately 1-2 mutations per month. ${ }^{3}$ Over the course of infection, the major SARS-CoV-2 population remains identical in most patients. ${ }^{3}$ The sudden appearance of a large cluster of non-synonymous substitutions of immunologic significance is therefore unusual. The prevailing theory is that VOC arose from prolonged SARS-CoV-2 shedding in immunocompromised patients. Although live viruses have not been cultured in the majority of those infected after day 9 of symptom onset or initial PCR positivity on nasopharyngeal swab, those who are immunocompromised can exhibit prolonged viral replication, lasting up to 119 days. ${ }^{4,5}$ The addition of therapies which exert specific selection pressure on the virus in the context of an immunocompromised host may combine to accelerate SARSCoV-2 genomic evolution. Emergence of new dominant mutations in immunocompromised patients who receive corticosteroids, intravenous immunoglobulins, monoclonal antibodies and convalescent plasma are being increasingly reported - although it remains unclear whether the enhanced diversity in these patients is due to uninhibited replication or specific selection pressures from treatment. 6,7

\section{Will the new variant increase transmission?}

It is important to investigate whether changes in virus phenotype would lead to meaningful increases in transmission between humans. Epidemiological analyses have suggested this to be the case for VOC. For example, whole genome sequence data collected from community-based diagnostic testing showed an increase in prevalence of VOC over September to December 2020 at an exponential rate. Estimated reproduction numbers for VOC are substantially higher than for non-VOC lineages, with an estimated overall difference of $0.4-0.7 .^{8}$ Independent two-strain 
mathematical models of SARS-CoV-2 transmission fitted to the prevalence of SARS-CoV-2, the relative frequency of VOC, observed COVID-19 hospital admissions, hospital, intensive care unit (ICU) bed occupancy and deaths from COVID-19 in the most heavily affected regions in the UK also suggest a $56 \%$ increase in transmission (95\% credible interval 50-74\%) compared to other variants. ${ }^{9}$

These estimates are of particular concern because they were calculated during periods when many regions of the UK were in the highest social distancing restrictions (Tier 4, Table 1). The previous UK tiered system is therefore insufficient to contain VOC. Despite the virus having infected over 4 million and killed over 120 thousand people in the UK, the majority of the population are not immune to SARS-CoV-2. The new variant poses a major risk of rapid spread to vulnerable populations, such as those with cardiovascular comorbidities, ethnic minority groups or the elderly. ${ }^{10,11}$ The implementation of a third national lockdown on 5 January 2021 aimed to address the same problem that all countries faced this time last year, at the start of the pandemic - to prevent excess deaths that would result when all reasonable capacity in hospital is exceeded.

However, all levels of meaures implemented over the past year in the UK still allowed a degree of social mixing and close contact indoors, for example in key worker groups such as bus drivers, educational settings and places of worship (highlighted in bold in Table 1). Clusters of positive cases arising from these settings are a key characteristic of SARS-CoV-2 transmission. For instance, an outbreak investigation from China identified that 24 out of 67 passengers were infected during a 50 -minute return bus journey, which was linked to an index case who was symptomatic the day before the trip. ${ }^{12}$ In Washington State, a mildly symptomatic index case attended a choir practice lasting 2.5 hours: out of 61 persons, 32 confirmed and 20 probably secondary COVID-19 cases occured, with an attack rate of $53.5 \%$ to $86.7 \% .{ }^{13}$ In November and December 2020, educational settings were among the largest institutions in the UK linked to SARS-CoV-2 infections, which remained open during Tier 4 measures and is most implicated in viral transmission (a significantly larger proportion of VOC cases has been reported in under 20 year olds than non-VOC cases). The only way to prevent viral transmission is to stop its ongoing replication in a susceptible population; this must be communicated effectively to the public in the current lockdown, and is likely to be the major reason why the draconian measures undertaken in East Asia (where the population had been primed by previous experiences with SARS) were effective at suppressing subsequent waves of infection. Any population mixing will mean ongoing viral replication, with likely ongoing implications on those with longterm health conditions and the national economy.

\section{Ongoing consequences of the variant}

The rise of new variants in the community also poses significant challenges to the contact tracing efforts that will be needed to effectively maintain control of the virus locally once lockdown measures end, until population-level immunity is achieved. In this regard, the current national Track and Trace system, which places emphasis on a centralised contact tracing platform and does not take into account the local needs of the community, has proved to be insufficient. Increased efforts must be made to work with local public health authority and health services, who are best placed to understand the needs of their areas of jurisdiction, and to implement both context- and area-specific interventions. Within Leicester, for example, where there is a diverse ethnic population, this would mean establishing relationships with community leaders to create a bespoke, culturally appropriate interventions that these high-risk communities can easily access.

VOC also has consequences for the UK vaccination programme. A more transmissible virus will require greater vaccine uptake for population-level control. Recently, the Joint Commitee on Vaccination and Immunisation (JCVI) recommended prolongation of the second dose of both the Pfizer BioNTech and Oxford AstraZeneca vaccines to 12 weeks, to increase vaccine uptake amongst the most vulnerable. Vaccines are typically given in more than one dose to increase the quantity, quality and longevity of antibody responses. The Oxford/AstraZeneca vaccine demonstrated efficacy at a range of dose intervals, with antibody responses after the boost being signicantly stronger with longer intervals. ${ }^{14}$ For the Pfizer BioNTech vaccine, efficacy in the period from 14 days after first dose to 21 days is high. ${ }^{15}$ Although there is no direct evidence of efficacy beyond a 3-week interval, there is also no evidence in existing vaccines for a stronger immunological response having been exerted at 3 weeks compared to longer intervals. It is therefore a pragmatic intervention recommended based on first principles and experience from previous vaccines, in the absence of high-quality evidence, with the aim of maximising protection in the population.

Finally, the presence of VOC is already global. Other highly transmissible variants, such as one identified in South Africa, have appeared. There is now an urgent need to determine whether these lineages could affect the efficacy of vaccines, since all current approved vaccines aim to construct the $S$ protein. Theoretically the impact should be minimal, as the S protein will have many epitopes as immunological targets. However, should the mutation affect protein folding in the new variant, this may affect binding of antibodies induced by the vaccine to older variants. There is also the possibility that novel variants may escape vaccine control, particularly in the context of a partially immunised population, which may in turn exert a strong selection pressure on the SARSCoV-2. Vaccine developers will need to consider experimenting with variant sequences and powering post-licensing studies to detect differences in transmission and efficacy between preexisting and new variants. Licensing authorities may also need to clarify abbrieviated pathways to marketing for vaccines that involve altering strain formulation, without any other changes to their composition - similar to what is in place for the annually updated seasonal influenza vaccine.

\section{Conclusion}

The arrival of VOC marks a new chapter in the global fight against COVID-19, and with it, new challenges that must be addressed It is likely that, at least for now, we will have to learn to live with the virus.

\section{References}

1 Public Health England. Investigation of novel SARS-COV-2 variant: Variant of Concern 202012/01. PHE, 2020. https://assets.publishing. service.gov.uk/government/uploads/system/uploads/attachment_ data/file/959438/Technical_Briefing_VOC_SH_NJL2_SH2.pdf.

2 Public Health England. Investigation of novel SARS-CoV-2 variant: Variant of Concern 202012/01: Technical briefing 2. PHE, 2020. 


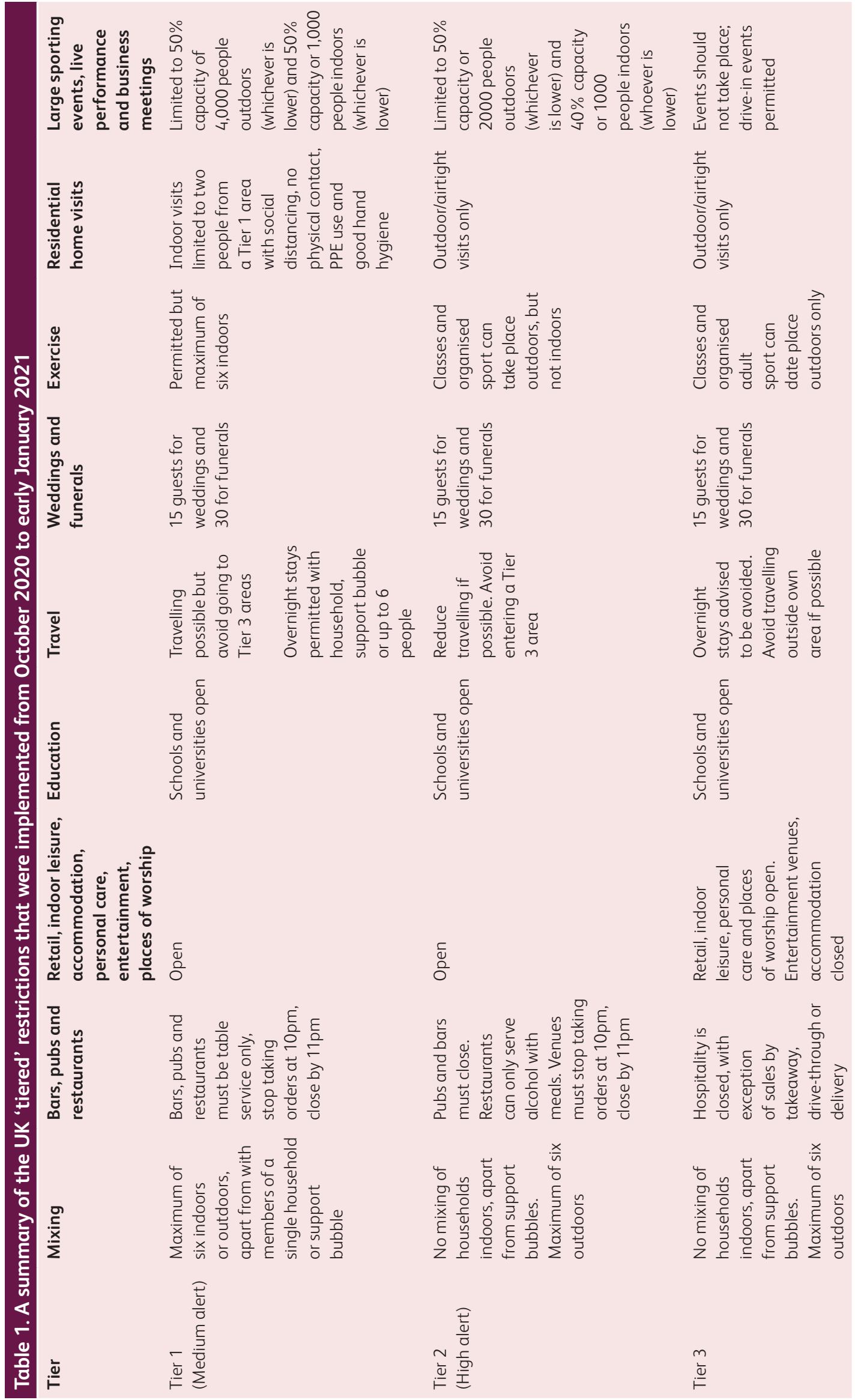




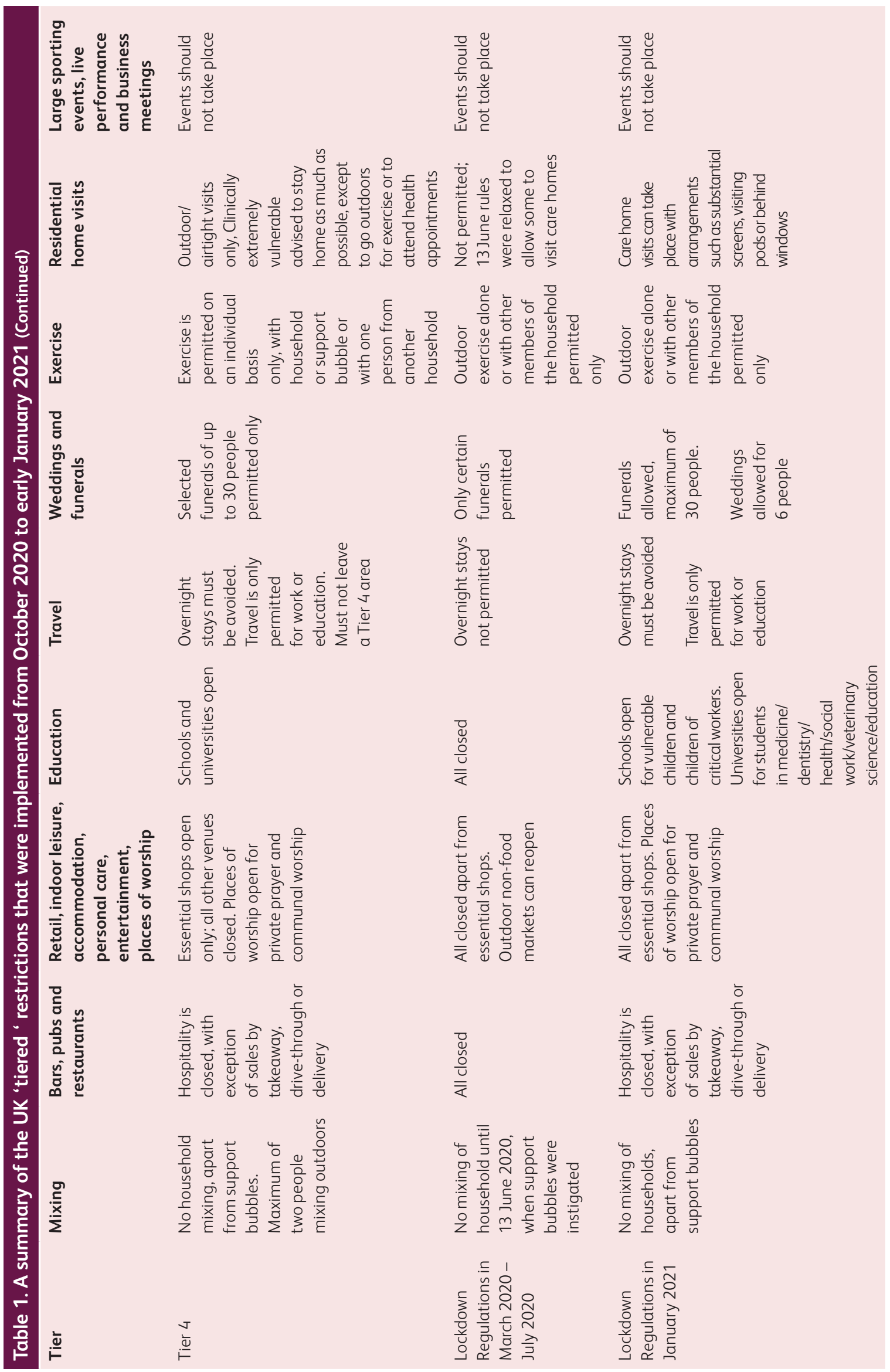


https://assets.publishing.service.gov.uk/government/uploads/ system/uploads/attachment_data/file/959438/Technical_Briefing_ VOC_SH_NJL2_SH2.pdf.

3 Duchene S, Featherstone L, Haritopoulou-Sinanidou M et al. Temporal signal and the phylodynamic threshold of SARS-CoV-2. Virus Evol 2020;6:veaa061.

4 Baang JH, Smith C, Mirabelli C et al. Prolonged severe acute respiratory syndrome coronavirus 2 replication in an immunocompromised patient. J Infect Dis 2020;223:23-7.

5 Cevik M, Tate M, Lloyd O et al. SARS-CoV-2, SARS-CoV, and MERS-CoV viral load dynamics, duration of viral shedding, and infectiousness: a systematic review and meta-analysis. Lancet Microbe 2021;2:e13-e22.

6 Choi B, Choudhary MC, Regan ] et al. Persistence and evolution of SARS-CoV-2 in an immunocompromised host. N Engl ] Med 2020;383:2291-3.

7 Avanzato VA, Matson M], Seifert SN et al. Case study: prolonged infectious SARS-CoV-2 shedding from an asymptomatic immunocompromised individual with cancer. Cell 2020;183:1901-12.

8 Volz E, Mishra S, Chand M et al. Transmission of SARS-CoV-2 Lineage B.1.1.7 in England: Insights from linking epidemiological and genetic data. MedRxiv 2020; 2020.12.30.20249034.

9 Davies N, Barnard R, Jarvis C et al. Estimated transmissibility and severity of novel SARS-CoV-2 variant of concern 202012/01 in England. MedRxiv 2020; 2020.12.24.20248822.

10 Sze S, Pan D, Nevill C et al. Ethnicity and clinical outcomes in COVID-19: A systematic review and meta-analysis. EClinicalMedicine 2020;29:100630
11 Huang C, Wang Y, Li X et al. Clinical features of patients infected with 2019 novel coronavirus in Wuhan, China. Lancet 2020;395: 497-506.

12 Shen Y, Li C, Dong H et al. Community outbreak investigation of SARS-CoV-2 transmission among bus riders in Eastern China. JAMA Intern Med 2020;180:1665-71.

13 Hamner L, Dubbel P, Capron I et al. High SARS-CoV-2 attack rate following exposure at a choir practice. Morb Mortal Wkly Rep High 2020;69:606-10.

14 Medicines and Healthcare Products Regulatory Agency. Information for healthcare professionals on COVID-19 vaccine AstraZeneca. UK Government, 2020. Available from: www.gov.uk/ government/publications/regulatory-approval-of-covid-19-vaccineastrazeneca/information-for-healthcare-professionals-on-covid-19vaccine-astrazeneca.

15 Polack FP, Thomas SJ, Kitchin N et al. Safety and efficacy of the BNT162b2 mRNA Covid-19 vaccine. N Engl J Med 2020;383: 2603-15.

Address for correspondence: Dr Manish Pareek, Department of Respiratory Sciences, Maurice Shock Medical Sciences Building, University Road, Leicester LE1 9HN, UK.

Email: manish.pareek@leicester.ac.uk

Twitter: @drmanpareek 